\title{
Medial temporal lobe volume predicts rate of learning in Rey-AVLT
}

\author{
Sven-Erik Fernaeus*, Per Julin, Ove Almqvist, Lars-Olof Wahlund \\ ${ }^{1}$ Department of Neurobiology, Care Sciences and Society, Karolinska Institute, Stockholm, Sweden; \\ *Corresponding Author: fernaeus@mac.com
}

Received 8 December 2012; revised 20 January 2013; accepted 29 January 2013

\begin{abstract}
The medial temporal lobe (MTL) has been assigned a central role in human episodic memory and learning. Evidence for this comes from PET and fMRI studies as well as lesion studies. This study aimed at comparing the effect of atrophy at repeated trials of a supraspan test of memory. Included in the study were patients with Alzheimer's Disease, Mild Cognitive Impairment, and Subjective Memory Disorders as well as Controls $(n=116)$. The supraspan test used was the Rey Auditory Verbal Learning test (RAVLT). Comparisons between extreme groups with high (Stanine 6 - 9) and low (Stanine 1 - 4) intracranial proportions (IP) of MTL were made at the five trials of RAVLT. There was a significantly higher rate of learning among subjects with high MTL IP compared to those with low MTL IP in both hemispheres. There was no difference in the rate of list learning performance due to education or age and interestingly: the list learning rates among subjects with high/low Lateral Temporal Lobe IPs were almost similar. The hemispheric differences regarding learning rate were small and insignificant. Results are discussed in terms of hippocampal involvement in associative processes necessary in supraspan list learning.
\end{abstract}

Keywords: Hippocampus; Learning; Medial Temporal Lobe; Episodic Memory; MRI; Atrophy

\section{INTRODUCTION}

The medial temporal lobe (MTL) including the hippocampus has been tied to one or all of the main episodic memory functions: encoding, storage and/or retrieval. Different views regarding this function of MTL have been proposed. One theory, the encoding and retrieval hypothesis, holds that the hippocampal system binds different components of an event by linking simultane- ous activation in distributed brain regions [1-4]. According to this theory hippocampus should be activated both during encoding and retrieval. Another theory is the novelty assessment hypothesis [5-7]. According to this theory hippocampus is involved primarily as a novelty detector, which is engaged only when entirely new events are encoded. Consequently both of these theories implicate hippocampal involvement in learning new associations.

Although there has been a long history of research concerning lesions of the medial temporal lobe (MTL) and its connection to amnesia [8-12] and in spite of the growing body of brain imaging research regarding the connection between MTL and human memory in the last decade, very few successful attempts have been made to link MTL directly to the rate of learning [13]. Regarding the relationship between episodic memory and MTL functioning, a number of imaging studies in fact failed to show any encoding related MTL activations [14-18]. The reason for the failure to detect activation of hippocampus during learning may have methodological reasons due to the relatively small regions that are involved, less than $5 \%$ of the intracranial volume, and the low time resolution especially regarding the PET studies.

On the other hand, when looking at different aspects of episodic memory, such as encoding or retrieval, recent activation studies have revealed encoding or retrieval related activations [19-22]. Furthermore, posterior regions of hippocampus or parahippocampal regions have shown activation during visual associative encoding, associative learning and successful verbal encoding [23-25]. When, in activation studies, a more precise selection of region of interest within MTL is made, the lesion findings regarding the critical role of hippocampus in associative learning or episodic memory may thus be confirmed.

Soininen et al. explicitly investigated the relationship between MTL volumes and memory function among subjects with age associated memory impairment and controls [26]. They found a positive correlation between episodic memory function and volumes of amygdala/ 
hippocampus.

To test the hypothesis that hippocampus is related to rate of learning, a study by Jones-Gotman et al. was made with 40 controls and 71 MTL lesion cases taken from three different countries [13]. In that study an effect on memory for earlier learned words was found, but the rate of word list learning was not clearly related to MTL lesions. Jones-Gotman et al. proposed broken connections between hippocampus and temporal neocortex to be responsible for the lower level of learning and the impaired memory in their MTL lesion patients, in line with hypotheses presented by Larry Squire [27].

The aim of the present study was to compare subject with MTL and TL atrophy with subjects with little or no atrophy in these regions regarding their supraspan word list learning ability.

\section{METHOD}

\subsection{Participants}

To get a sample of participants with sufficiently varying degrees of memory impairment and MTL atrophy all consecutive patients with Subjective Memory Disorders (SMD), Mild Cognitive Impairment (MCI) or Alzheimer's disease (AD) in addition to a group of healthy elderly subjects (HC) who had taken the Rey-AVLT prior to the study at Huddinge University Hospital were selected. Comparisons between these diagnoses will be reported in a separate study.

\subsection{Procedure}

All participants had taken the Rey Auditory Verbal Learning Test (RAVLT) as part of a routine neuropsychological examination procedure [28]. All these participants also underwent a Magnetic Resonance Imaging examination, and volumetric measures were obtained through the stereologic method [29].

\subsection{Division of Subjects into Extreme Groups}

In order to minimize errors of measurement partici- pants were divided into extreme groups on the basis of volumetric measures. This division was based on a Stanine routine. Scores 0.25 standard deviations below or above the mean score were thus considered Low or High in respect of their volumetric intracranial proportion for each of four measures: Right or Left Medial or Lateral Temporal Volume proportion of intracranial volume. There were no differences in education $(F<1)$ between any of these four divisions and no significant differences in age, see Table 1.

\section{RESULTS}

\subsection{Effects of Volumes on RAVLT Scores}

There was no significant effect of Right Lateral Temporal Lobe (RTL) proportion on RAVLT scores $(F<1)$ and there was no significant interaction between trials and RTL volume, Rao $R(4,81)=0.26, p=0.90$, When age and education were entered as covariates, there were no significant within cells regressions, Rao R Form 3 (10, $158)=0.97, p=0.47$, see Figure 1.

There was a tendency of an LTL effect on RAVLT scores, $F(1,80)=3.75, p=0.06$, but there was no interaction between trials and LTL volume, Rao $R(4,67)=$ $0.25, p=0.91$, see Figure 2. When age and education were entered as covariates, there were no significant within cells regressions, Rao R Form $3(10,180)=1.32$, $p=0.22$, see Figure 3 .

There was a significant effect of Right Medial Temporal Lobe volume (RMTL) on RAVLT scores, $F(1,81)$ $=8.01, p<0.01$, but there was also a significant interacttion between RMTL and Trials, Rao $R(4,78)=4.01, p<$ 0.01 . When age and education were entered as covariates, there were no significant within cells regressions, Rao $R$ Form $3(10,188)=1.26, p=0.26$, see Figure 2.

There was a significant effect of Left Medial Temporal Lobe (LMTL) volume on RAVLT scores, $F(1,79)=$ $3.98, p<0.05$ and there was also a significant interaction between LMTL and RAVLT scores, Rao $R(4,73)=3.60$, $p<0.01$. When age and education were entered as covariates, there were no significant within cells regressions, Rao R Form $3(10,124)=1.21, p=0.29$, see Figure 4.

Table 1. Mean age, education and number of cases in each of the four divisions based on right and left medial or lateral temporal lobe proportion of intracranial volume.

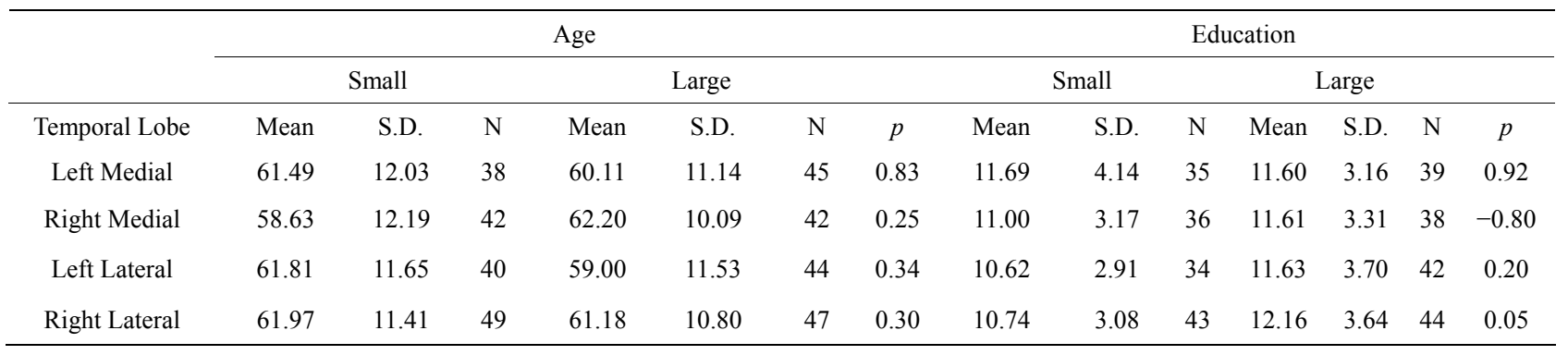




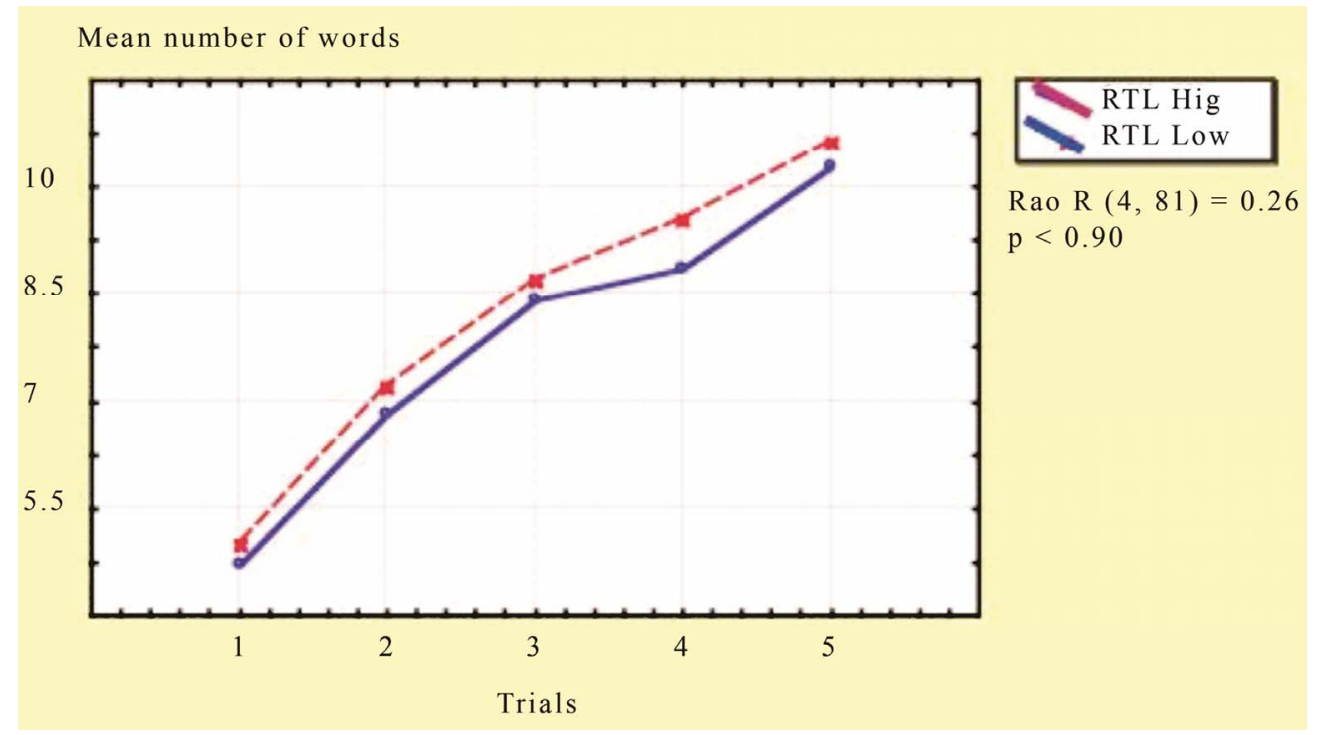

Figure 1. AVLT list learning in RTL-low (blue) and RTL-high (red) IPs.

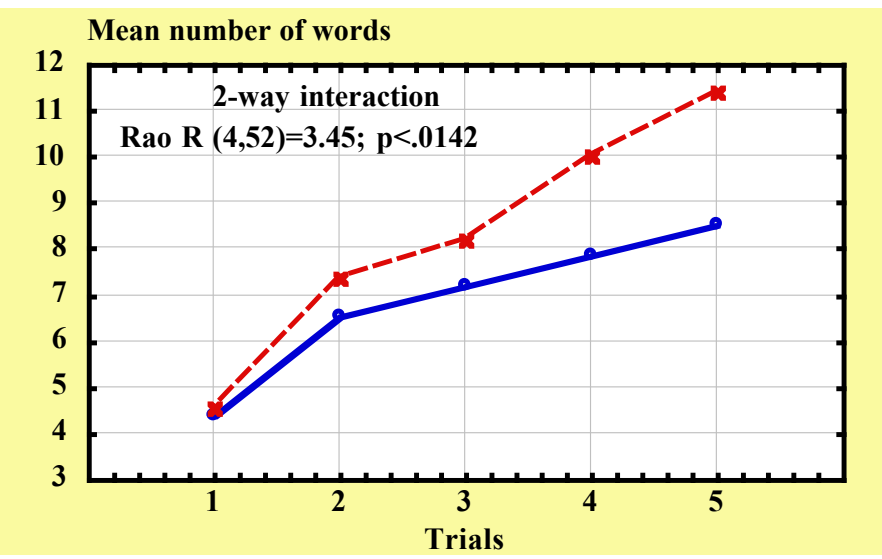

Figure 2. AVLT list learning in RMTL-low (blue) and RMTL-high IPs.

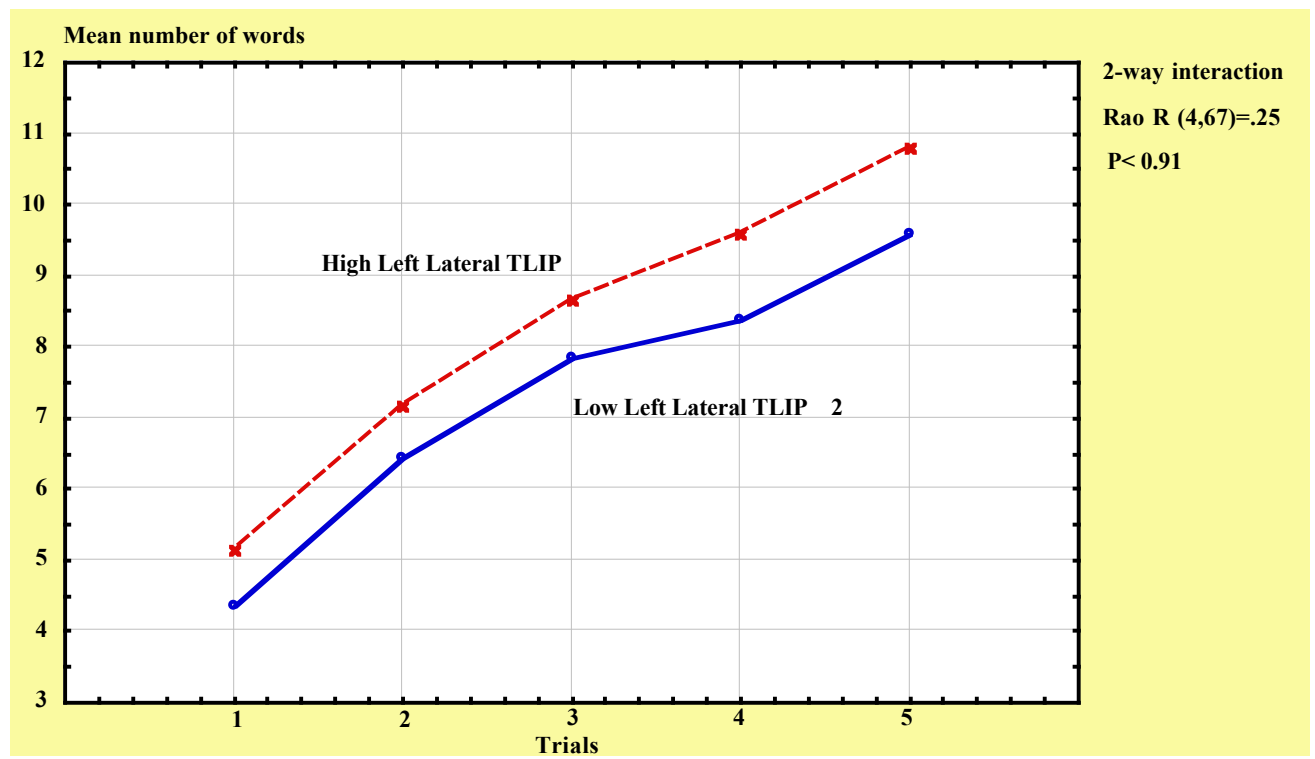

Figure 3. Rey-AVLT learning among subjects with high/low left lateral temporal intracranial proportion. 


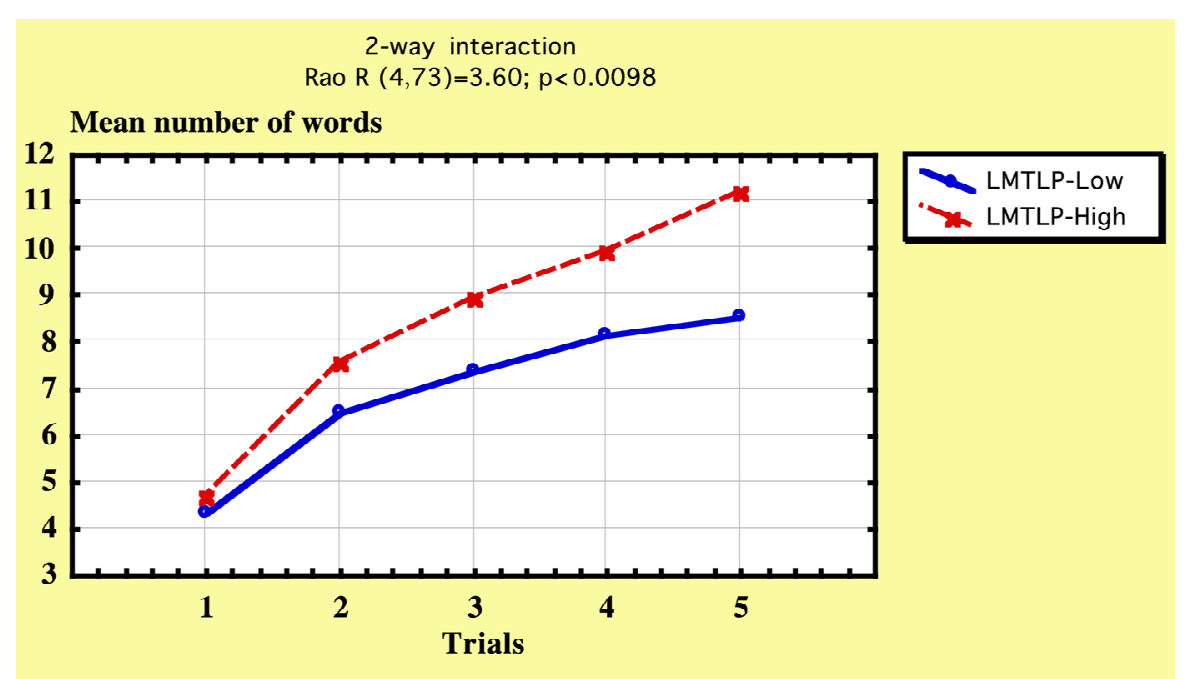

Figure 4. AVLT list learning in LMTL-low (blue) and LMTL-high IPs.

\subsection{Educational and Age Effects}

There were no significant within cell regressions due to age or education in the analyses of volumetric effects on RAVLT scores across trials. There was, however, one significant difference in educational level between the two divisions based on Right Lateral Temporal Lobe proportion (RTL), as can be seen in Table 1. To check for possible differential effects across trials, the within cell regressions were calculated at each trial. In the division based on RTL there were significant within cell regressions in one trial (trial 2) of RAVLT, F (2, 98) $=4.07$, $\mathrm{p}<0.05$, with a significant contribution from Education, $\mathrm{b}=0.20, \mathrm{t}=2.56, \mathrm{p}<0.05$.

\section{DISCUSSION}

In this study we compared participants with relatively high and low intracranial proportion of MTL volume (MTL IP) in their performance across the five trials of the Rey-AVLT. There was an interaction between trials and MTL IP division indicating a higher word list learning rate among subjects with high MTL IP compared to participants with low MTL IP. The interaction could not be attributed to a higher educational level among subjects with higher learning rate, nor could it be explained by a higher age among subjects with atrophied MTL. We also found that the same type of division based on Lateral Temporal Lobe did not discriminate learning rates, the interaction between trials and different intracranial proportions of lateral temporal lobe was nonexistent.

These results are not unexpected due to what is hitherto known about the critical role played by the hippocampal region in episodic memory encoding and associative learning [23-25]. Nor are these results unexpected due to what is known about learning disabilities and medial temporal lobe atrophy among Alzheimer's disease patients [30]. Nevertheless our results may give further support to the hypothesis of the medial temporal lobe as a learning structure quite dissociable in this respect from the neocortical lateral temporal lobe. The nonexistent interaction between trials and LTL IP in each hemisphere is a clear indicator of non-involvement on behalf of the lateral temporal lobe in supraspan list learning.

Measurement errors regarding the independent variable may be critical in an experiment of this kind and amount to about $5 \%$ in volumetric measurements based on the stereologic method [29]. In such cases caution should lead us to relatively broad border lines between groups to be compared on the basis of such a variable. Broad borderlines were used in this study in order to erase possible classification errors. We therefore excluded all subjects with stanine 5 in the four measures of MTL and LTL intracranial proportions. In so doing we probably ensured a clear division between high and low MTL or LTL intracranial proportions. Because stanine divides the sample in segments one-half standard deviation in width, we can be relatively sure that classification errors due to measurement error are minimized if not excluded.

Turning to the theoretical implications to be drawn from this study, we would rather see the incremental learning from trial to trial as a buildup of associative links between the items of the supra span list of ReyAVLT. All words in the list are concrete high imagery nouns. Neighbouring words may be easy to associate for a normal learner, who may visualize groups of objects, which the nouns refer to, into imagery scenes. Interpretating the learning progress in the Rey-AVLT this way leads most likely to an explanation of our findings that would be in line with those who regard hippocampus as an associative device primarily involved in establishing new associations [23-25]. 
The supraspan list learning test may thus be seen as a test of the ability to form new associations between neighbouring words in the list. But we must also take into account the general association between all the words and the list itself. This may be formulated as the prime problem of the task. Each word, attended to and hopefully understood, must be given a new property: "to belong to the list that is repeated". This new knowledge will be gained through the repeated possibilities to establish new links between the words. If a test person is successful in this elaboration of the list items during or after the five trials, the words in the supraspan list all will have a new relatively unique but probably less useful property outside the clinic: "to belong to the Rey AVLT word list". This type of knowledge is similar to the knowledge that Naftali and Gad belong to the list of Jacob's sons with the difference that the words in Rey AVLT are more frequent. If a person asked a modern psychologist to tell everything that he/she knows about any of the words belonging to the Rey AVLT list, the property of belonging to the Rey AVLT list would certainly be mentioned-with a laugh or hesitantly.

Regarding the MTL and LTL relative contribution to learning our results are almost but not entirely in agreement with the Jones-Gotman et al. study [31]. They did not report any significant interactions specifically between trials and lesion groups, but from graphs presented (p. 969) it seems as if their left amygdalohippocampectomy group exposes another type of learning curve compared to controls and their right amygdalohippocampectomy group. Regarding design list learning in the same MTL lesions groups, the learning curves deviate from controls already from trials two and three, but due to ceiling effects further learning rate effects are not seen. Because in the Rey-AVLT 15 items are studied, while in the Jones-Gotman et al. study only 13 items were used both for the word list and the design list, ceiling effects are less pronounced in our study.

If both MTL and the Lateral Temporal lobe (LTL) were involved in learning we would probably see an additive effect from atrophy in both regions on rate of learning. However, due to the significant interaction between trial and performance only for the medial temporal lobe, no such interpretation can be made. If we only see effects on learning rate from MTL from atrophy, but not from LTL, we would conclude that MTL is necessary for learning while LTL is necessary for some other function, but not necessarily for learning. Because we did not see any word list learning rate effects from LTL IPs $(p \approx$ $0.90)$, the conclusion is straightforward. Jones-Gotman et al. make a point, however, that must be considered. The common words represent well-established concepts in the lexicon of each subject taking the Rey-AVLT. Words are thus never really new as items to the word list learner, the only novelty of word lists used in testing is the new associations between the words that must be created to be produced above immediate span level. The neocortical lesion effect that Jones-Gotman et al. sees for designs, but not for words, may thus be attributed to a novelty detection mechanism being activated for items in design list learning. This novelty detection effect is also clearly demonstrated in the Jones-Gotman et al. study because it occurs between the first and the second trial, where learning curves are not parallel. The novelty detection effect may thus not be related to learning rate, because then the learning curves would differ more in later trials. Novelty detection would thus rather be an episodic memory phenomenon, not a clear cut learning phenomenon and it may be attributed to the Jones-Gotman et al. hypothesis of the cooperation between MTL and LTL.

\section{CONCLUSION}

Our study gives further support to the associative-encoding hypothesis regarding medial temporal lobe functioning [23-25].

\section{REFERENCES}

[1] Squire, L. and Zola-Morgan, S. (1991) The medial temporal lobe memory system. Science, 253, 1380-1386. doi:10.1126/science. 1896849

[2] Squire, L. (1992) Memory and the hippocampus: A synthesis from findings with rats, monkeys and humans. Psychological Review, 99, 195-231. doi:10.1037/0033-295X.99.2.195

[3] Cohen, N.J. and Eichenbaum, H. (1993) Memory, amnesia, and the hippocampal system. MIT Press, Cambridge.

[4] Eichenbaum, H., Otto, T. and Cohen, N.J. (1994) Two functional components of the hippocampal memory system. Behavioural \& Brain Science, 17, 449-518.

[5] Tulving, E., Kapur, S., Craik, F., Moscovitch, M. and Houle, S. (1994) Hemispheric encoding/retrieval asymmetry in episodic memory: Positron emission findings. Proceedings of the National Academy of Sciences of the United States of America, 91, 2016-2020. doi:10.1073/pnas.91.6.2016

[6] Tulving, E., Markowitsch, H.J., Craik, F.I.M., Habib, R. and Houle, S. (1996) Novelty and familiarity activations in PET studies of memory encoding and retrieval. Cerebral Cortex, 6, 71-79. doi:10.1093/cercor/6.1.71

[7] Knight, R.T. (1996) Contribution of human hippocampal region to novelty detection. Nature, $\mathbf{3 8 3}, 256-259$. doi: $10.1038 / 383256 \mathrm{a} 0$

[8] Scoville, W. and Milner, B. (2000) Loss of recent memory after bilateral hippocampal lesions. Journal of $\mathrm{Neu}$ ropsychiatry Clinical Neuroscience, 12, 103-113.

[9] Penfield, W. and Milner, B. (1958) Memory deficit produced by bilateral lesions in the hippocampal zone. $A M A$ Archives of Neurology and Psychiatry, 79, 475-497. 
doi:10.1001/archneurpsyc.1958.02340050003001

[10] Zola-Morgan, S., Squire, L.R. and Amaral, D.G. (1986) Human amnesia and the medial temporal region: Enduring memory impairment following a bilateral lesion limited to field CA1 of the hippocampus. Journal of Neuroscience, 6, 2950-2967.

[11] Victor, M. and Agamanolis, D. (1990) Amnesia due to lesions confined to the hippocampus: A clinical-pathologic study. Journal of Cognition Neuroscience, 2, 246-257. doi:10.1162/jocn.1990.2.3.246

[12] Rempel-Clower, N., Zola, S., Squire, L. and Amaral, D. (1996) Three cases of enduring memory impairment after bilateral damage limited to the hippocampal formation. The Journal of Neuroscience, 16, 5233-5255.

[13] Jones-Gotman, M., Zatorre, R.J., Olivier, A., Andermann, F., Cendes, F., Staunton, H., McMackin, D., Siegel, A. and Wieser, H.-G. (1997). Learning and retention of words and designs following excision from medial or lateral temporal-lobe structures. Neuropsychologia, 35, 963-973. doi:10.1016/S0028-3932(97)00024-9

[14] Kapur, S., Craik, F.I.M., Tulving, E., Wilson, A.A., Houle, S. and Brown, G.M. (1994) Neuroanatomical correlates of encoding in episodic memory: levels of processing effect. Proceedings of the National Academy of Sciences of the United States of America, 19, 2008-2011. doi:10.1073/pnas.91.6.2008

[15] Kapur, S., Tulving, E., Cabeza, R., McIntosh, A.R., Houle, S. and Craik, F.I.M. (1996) Neural correlates of intentional learning of verbal materials: A PET study in humans. Cognitive Brain Research, 4, 243-249. doi:10.1016/S0926-6410(96)00058-4

[16] Nyberg, L., McIntosh, A.R., Houle, S., Nilsson, L.G. and Tulving, E. (1996) Activation of medial temporal structures during episodic memory retrieval. Nature, 25, 669670.

[17] Shallice, T., Fletcher, P., Frith, C.D., Grasby, P., Frackowiak, R.S.J. and Dolan, R.J. (1994) Brain regions associated with aquisition and retrieval of verbal episodic memory. Nature, 368, 633-635. doi:10.1038/368633a0

[18] Tulving, E., Kapur, S., Craik, F.I.M., Moscovitch, M. and Houle, S. (1994) Hemispheric encoding/retrieval asymmetry in episodic memory: Positron emission tomography findings. Proceedings of the National Academy of Sciences of the United States of America, 91, 2016-2020. doi:10.1073/pnas.91.6.2016

[19] Gabrieli, J.D.E., Brewer, J.B., Desmond, J.E. and Glover, G.H. (1997) Separate neural bases of two fundamental memory processes in the human medial temporal lobe. Science, 276, 264-266. doi:10.1126/science.276.5310.264

[20] Grady, C.L., McIntosh, A.R., Horwitz, B., Maisog, J.M., Ungerleider, L.G., Mentis, M.J., Pietrini, P., Schapiro, M.B. and Haxby, J.V. (1995) Age-related reductions in human recognition memory due to impaired encoding. Science, 269, 218-221. doi:10.1126/science.7618082

[21] Stern, C.E., Corkin, S., Gonzales, R.G., Guimares, A.R., Baker, J.R., Jennings, P.J., Carr, C.A., Sugiura, R.M., Ve- dantham, V. and Rosen, B.R. (1996) The hippocampal formation participates in novel picture encoding: Evidence from functional magnetic resonance imaging. Proceedings of the National Academy of Sciences of the United States of America, 93, 8660-8665.

doi:10.1073/pnas.93.16.8660

[22] Tulving, E., Kapur, S., Markowitsch, H.J., Craik, F.I.M., Habib, R. and Houle, S. (1994) Neuroanatomical correlates of retrieval in episodic memory: Auditory sentence recognition. Proceedings of the National Academy of Sciences of the United States of America, 91, 2012-2015. doi:10.1073/pnas.91.6.2012

[23] Fernandez, G., Weyerts, H., Schrader-Bölsche, M., Tendolkar, I., Smid., H.O.M., Tempelmann, C., Hinrichs, H., Schein, H., Elger, C.E., Mangun, G.R. and Heinze, H.-J. (1998) Successful verbal encoding into episodic memory engages the posterior hippocampus: A parametrically analyzed functional magnetic resonance imaging study. The Journal of Neuroscience, 18, 1841-1847.

[24] Henke, K., Buck, A., Weber, B. and Wieser, H.G. (1998) Human hippocampus establishes associations in memory. Hippocampus, 7, 249-256.

doi:10.1002/(SICI)1098-1063(1997)7:3<249::AID-HIPO 1>3.0.CO;2-G

[25] Rombouts, S.A.R.B., Machielsen, W.C.M., Witter, M.P., Barkhof, F., Lindeboom, J. and Scheltens, P. (1997) Visual association encoding activates the medial temporal lobe: A functional magnetic resonance imaging study. Hippocampus, 7, 594-601.

doi:10.1002/(SICI)1098-1063(1997)7:6<594::AID-HIPO 2>3.0.CO;2-F

[26] Soininen, H.S., Partanen, K., Pikänen, A., Vainio, P., Hänninen, T., Hallikainen, M., Koivisto, K. and Riekkinen, P.J. (1994) Volumetric MRI analysis of the amygdala and the hippocampus in subjects with age-associated memory impairment: Correlation to visual and verbal memory. Neurology, 44, 1660-1668. doi:10.1212/WNL.44.9.1660

[27] Squire, L.R. (1992) Memory and the hippocampus: A synthesis from findings with rats, monkeys, and humans. Psychological Review, 99, 195-231. doi:10.1037/0033-295X.99.2.195

[28] Rey, A. (1964) L'exconen clinique en psychologie. Presses Universitaires de France, Paris.

[29] Julin, P., Melin, T., Andersen, C., Isberg, B., Svensson, L. and Wahlund, L.-O. (1997) Reliability of interactive threedimensional brain volumetry using MP-RAGE magnetic resonance imaging. Psychiatry Research, 76, 41-49.

[30] Almkvist, O. (1996) Neuropsychological features of early Alzheimer's disease: Preclinical and clinical stages. Acta Neurologica Scandinavica Supplement, 165, 63-71. doi:10.1111/j.1600-0404.1996.tb05874.x

[31] Jones-Gotman, M. and Milner, B. (1998) Right temporallobe contribution to image-mediated verbal learning. Neuropsychologia, 16, 61-71. doi:10.1016/0028-3932(78)90043-X 